\title{
Compensation of frequency independent I/Q imperfections using LMS algorithm
}

\begin{abstract}
Due to the increasing demand of high data rate in recent years, orthogonal frequency division multiplexing (OFDM) and Direct up conversion transmitters (DCT) have gained significant interest. However such system's efficiency can be compromised by front-end impairments such as In-phase Quadrature (I/Q) imperfections. In this paper the effects of IQ imbalance on transmitter is studied and an adaptive algorithm is developed to compensate such distortions. Simulation results show high level of accuracy in the proposed algorithm and its ability to estimate the IQ imperfections. It also shows that the proposed compensator could overcome the imperfections perfectly.
\end{abstract}

Keyword: Analog imperfections; OFDM; Up conversion; Gain and phase imbalance; LMS 